Document downloaded from:

http://hdl.handle.net/10251/46731

This paper must be cited as:

Gutiérrez, J.; Romaguera Bonilla, S.; Sanchis, M. (2012). Standard fuzzy uniform structures based on continuous t-norms. Fuzzy Sets and Systems. 195:75-89. doi:10.1016/j.fss.2011.10.008.

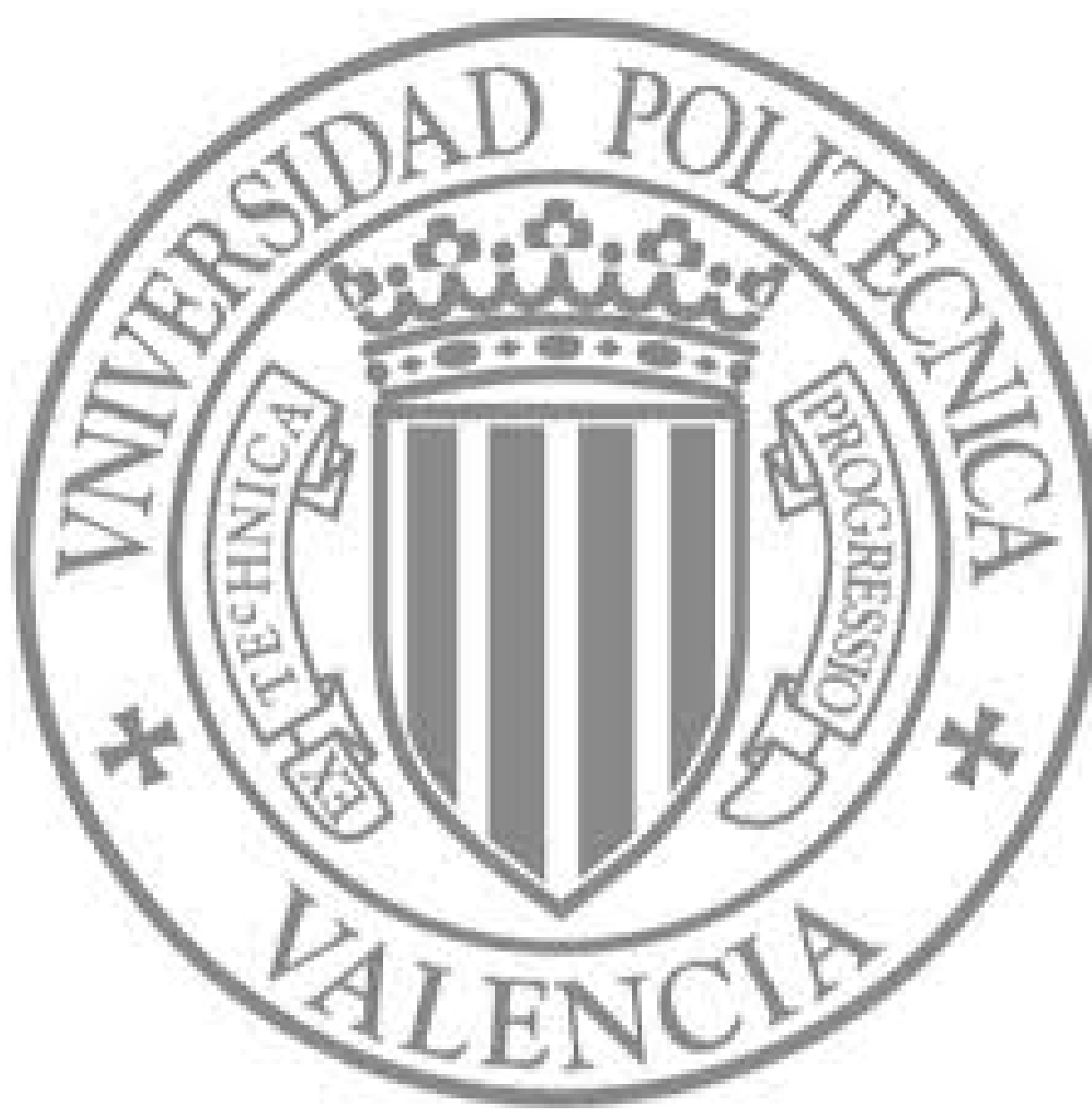

The final publication is available at

http://dx.doi.org/10.1016/j.fss.2011.10.008

Copyright Elsevier 


\title{
STANDARD FUZZY UNIFORM STRUCTURES BASED ON CONTINUOUS t-NORMS
}

\author{
J. GUTIÉRREZ-GARCÍA, S. ROMAGUERA, AND M. SANCHIS
}

\begin{abstract}
This paper deals with fuzzy uniform structures. Our approach involves a covariant functor $\Psi$ (introduced previously by the authors) from the category of fuzzy uniform spaces and fuzzy uniformly continuous mappings (in our sense) to the category of uniform spaces and uniformly continuous mappings. We show that $\Psi$ is well-behaved with respect to some significant fuzzy uniform concepts, which behavior provides a method to introduce notions of fine fuzzy uniform structure and Stone-Čech fuzzy compactification in this context. Our method also applies to obtain fuzzy versions of some classical results on topological algebra and hyperspaces. The case of quasi-uniform structures is also analyzed.
\end{abstract}

\section{INTRODUCTION}

In this paper we deal with a notion of fuzzy uniform space introduced by the authors in [13]. They proved in this previous paper that, for each continuous tnorm, the category of all fuzzy uniform spaces in authors' sense (and fuzzy uniformly continuous mappings) is isomorphic to the category of uniform spaces (and uniformly continuous mappings) by means of a covariant functor $\Psi$ which leaves mappings unchanged.

In this context, a question deserving further exploration is whether the functor $\Psi$ carries natural fuzzy uniform concepts to natural uniform concepts. A first step in this direction is presented in [13, Section 4] where it is shown that the image by $\Psi$ of a complete fuzzy uniform space is a complete uniform space. This fact permits us to show that a Hausdorff fuzzy uniform space has a unique Hausdorff fuzzy completion up to a fuzzy uniform isomorphism (see [13, Theorem 4.3 and Remark 4.4]).

In the light of the previous comments, the aim of the present paper is to reveal that the functor $\Psi$ behaves well with regard to certain fuzzy completions. The fact that $\Psi$ is well-behaved to respect to standard fuzzy uniform notions allows us to obtain fuzzy versions of some important results on topological algebra and hyperspaces. Although this procedure can be extended to take care of situations similar to the ones presented here, let us stress that the authors do not intend to do it. Extensions of our approach to other settings are left to the interested reader. For a contextualization of our approach in the realm of theories of fuzzified uniformities, see Section 1 and Remark 4.5 in [13].

The paper is organized as follows: in Section 2 we borrow from [13] the basic facts on fuzzy uniform structures that are useful in the sequel. Section 3 is devoted to discuss the fine fuzzy uniformity and the Stone-Cech fuzzy compactification (for

This research is supported by the Plan Nacional I+D+i, under grants MTM2009-12872-C02-01 and MTM2009-12872-C02-02. 
other different approaches to Stone-Čech type fuzzy compactifications see, for instance, $[12,18])$. Fuzzy uniform structures on topological groups and hyperspaces are studied in Section 4 and Section 5, respectively. Finally, in Section 6 we set up fuzzy quasi-uniform structures.

\section{Preliminaries AND NOTATiON}

Our basic references are [6] for general topology, and [9] and [14] for uniform spaces and uniform structures.

Next we recall some pertinent concepts on fuzzy (pseudo)metric spaces (in the sense of Kramosil and Michalek) which will be useful later on. We recall to the reader that in this context fuzzy means $[0,1]$-valued, where the usual order is taken on $[0,1]$.

A continuous t-norm [29] is a binary operation $*:[0,1] \times[0,1] \rightarrow[0,1]$ which satisfies the following conditions: $(\mathrm{i}) *$ is associative and commutative, (ii) $*$ is continuous, (iii) $a * 1=a$ for every $a \in[0,1]$, and (iv) $a_{1} * b_{1} \leq a_{2} * b_{2}$ whenever $a_{1} \leq a_{2}$ and $b_{1} \leq b_{2}$, with $a_{1}, a_{2}, b_{1}, b_{2} \in[0,1]$.

DEFINITION 2.1. [15] A fuzzy pseudometric on a set $X$ is a pair $(M, *)$ such that $M$ is a fuzzy set in $X \times X \times[0, \infty)$ and $*$ is a continuous t-norm satisfying for all $x, y, z \in X$ and $t, s>0$ :

(i) $M(x, y, 0)=0$;

(ii) $M(x, x, t)=1$;

(iii) $M(x, y, t)=M(y, x, t)$;

(iv) $M(x, y, t) * M(y, z, s) \leq M(x, z, t+s)$; and

(v) $M(x, y, \cdot):[0, \infty) \rightarrow[0,1]$ is left continuous.

If the fuzzy pseudometric $(M, *)$ satisfies (ii') $x=y$ if and only if $M(x, y, t)=1$ for all $t>0$, then $(M, *)$ is said to be a fuzzy metric $([15])$.

By a fuzzy (pseudo)metric space we mean a triple $(X, M, *)$ such that $X$ is a set and $(M, *)$ is a fuzzy (pseudo)metric on $X$.

Each fuzzy (pseudo)metric $(M, *)$ on $X$ induces a topology $\tau_{M}$ on $X$, which has as a base the family of open sets of the form $\left\{B_{M}(x, \varepsilon, t): x \in X, \varepsilon \in(0,1), t>0\right\}$, where $B_{M}(x, \varepsilon, t)=\{y \in X: M(x, y, t)>1-\varepsilon\}$ for all $x \in X, \varepsilon \in(0,1)$ and $t>0$.

In fact, every fuzzy (pseudo)metric $(M, *)$ induces a uniformity $\mathscr{U}_{M}$ on $X$ which has as a base the countable family $\left\{U_{n}: n \in \mathbb{N}\right\}$, where $U_{n}=\{(x, y) \in X \times X$ : $M(x, y, 1 / n)>1-1 / n\}$ for all $n \in \mathbb{N}$ (compare, for instance, [10, Theorem 1]). Hence the topology $\tau_{M}$ is (pseudo)metrizable.

Given two fuzzy pseudometrics $(M, *)$ and $(N, *)$ on $X$, the map $M \wedge N: X \times$ $X \times[0, \infty) \rightarrow[0,1]$ defined by $(M \wedge N)(x, y, t)=\min \{M(x, y, t), N(x, y, t)\}$ for each $x, y \in X$ and $t>0$ is a fuzzy pseudometric on $X$.

EXAMPLE 2.2. (Cf. $[8,10]$ ). Let $(X, d)$ be a (pseudo)metric space. Define a fuzzy set $M_{d}$ in $X \times X \times[0, \infty)$ by $M_{d}(x, y, 0)=0$, and $M_{d}(x, y, t)=t /(t+d(x, y))$ if $t>0$. Then $\left(M_{d}, \wedge\right)$ is a fuzzy (pseudo)metric on $X$, and thus $\left(M_{d}, *\right)$ is a fuzzy (pseudo)metric on $X$ for all continuous t-norm $*$, the so-called fuzzy (pseudo)metric induced by $(X, d)$, or the standard fuzzy (pseudo)metric of $(X, d)$. Note that the 
uniformities $\mathscr{U}_{d}$ and $\mathscr{U}_{M_{d}}$, induced, respectively, by $d$ and $\left(M_{d}, *\right)$, coincide $([13$, Proposition 2.5]), and thus the topologies induced by $d$ and $\left(M_{d}, *\right)$ are the same.

George and Veeramani introduced in [8] a modification of Kramosil and Michalek's notion of a fuzzy (pseudo)metric, taking fuzzy sets $M$ in $X \times X \times(0, \infty)$ with values in $(0,1]$, which satisfy conditions (ii) (resp. (ii')), (iii) and (iv) in Definition 2.1, and $\left(\mathrm{v}^{\prime}\right): M(x, y, \cdot):(0, \infty) \rightarrow(0,1]$ is continuous on $(0, \infty)$ for each $x, y \in X$.

Clearly, every fuzzy metric $(M, *)$ in George and Veeramani's sense can be considered as a fuzzy metric in Kramosil and Michalek's sense by putting $M(x, y, 0)=0$. Moreover, every standard fuzzy (pseudo)metric is actually a fuzzy (pseudo)metric in George and Veeramani's sense when we restrict $M_{d}$ to $X \times X \times(0, \infty)$.

The following concepts and results on fuzzy uniform structures and fuzzy uniform spaces may be found in [13].

DEFINITION 2.3. Let $X$ be a (nonempty) set and let $*$ be a continuous t-norm. A fuzzy uniform structure for $*$ is a nonempty family $(\mathscr{M}, *)$ of fuzzy pseudometrics $(M, *)$ on $X$ such that:

(FU1) if $(M, *),(N, *) \in(\mathscr{M}, *)$, then $(M \wedge N, *) \in(\mathscr{M}, *)$, and

(FU2) if $(M, *)$ is a fuzzy pseudometric on $X$, and if for each $\varepsilon \in(0,1)$ and each $t>0$, there exist $(N, *) \in(\mathscr{M}, *), \delta \in(0,1)$ and $s>0$ such that

$$
N(x, y, s) \geq 1-\delta \text { implies } M(x, y, t) \geq 1-\varepsilon
$$

for all $x, y \in X$, then $(M, *) \in(\mathscr{M}, *)$.

A fuzzy uniform structure $(\mathscr{M}, *)$ is called a Hausdorff fuzzy uniform structure if (FU3) for each $x, y \in X$ with $x \neq y$, there exist $(M, *) \in(\mathscr{M}, *)$ and $t>0$ such that $M(x, y, t)<1$.

By a (Hausdorff) fuzzy uniform space we mean a triple $(X, \mathscr{M}, *)$ such that $X$ is a set and $(\mathscr{M}, *)$ is a (Hausdorff) fuzzy uniform structure on $X$. If no confusion can arise, we will write $\mathscr{M}$ instead of $(\mathscr{M}, *)$.

For fuzzy uniform structures, the definitions of subbase and base are given in a similar way to the one in the case of uniform structures (see [13, Section 2]).

Clearly, each fuzzy uniform structure $(\mathscr{M}, *)$ on a set $X$ induces a topology $\tau_{\mathscr{M}}$ such that for each $x \in X$, the family $\left\{B_{M}(x, \varepsilon, t): M \in \mathscr{M}, \varepsilon \in(0,1), t>0\right\}$ is a neighborhood base at $x$. As in the realm of uniform spaces, a topological space $(X, \tau)$ is said to be fuzzy uniformizable if there is a fuzzy uniform structure $(\mathscr{M}, *)$ such that $\tau_{\mathscr{M}}=\tau$ and in this case, we say that $(\mathscr{M}, *)$ is admissible.

In Section 3 of [13] we define the category FUNIF of fuzzy uniform spaces and fuzzy uniformly continuous mappings, where a mapping $f:(X, \mathscr{M}, *) \rightarrow(Y, \mathscr{N}, \star)$ is said to be fuzzy uniformly continuous if for each $N \in \mathscr{N}, \varepsilon \in(0,1)$ and $t>0$ there exist $M \in \mathscr{M}, \delta \in(0,1)$ and $s>0$ such that $N(f(x), f(y), t)>1-\varepsilon$ whenever $M(x, y, s)>1-\delta$.

Furthermore, it is shown in Section 3 of [13] that given a t-norm $*$, the subcategory FUNIF $(*)$ whose objects are the fuzzy uniform spaces $(X, \mathscr{M}, *)$ is isomorphic to the topological category UNIF of uniform spaces (and uniformly continuous mappings) by means of a (covariant) functor which leaves mappings unchanged. 
This is done as follows: If $\mathscr{D}$ is a (Hausdorff) uniform structure on a set $X$, in the sense of [9], then the family $\{\{(x, y): d(x, y)<\varepsilon\}: d \in \mathscr{D}, \varepsilon>0\}$ is a base for a (Hausdorff) uniformity $\mathscr{U}_{\mathscr{D}}$ such that $\tau_{\mathscr{D}}=\tau_{\mathscr{U}}$. Conversely, if $\mathscr{U}$ is a uniformity on $X$, then the family $\mathscr{P}$ of all pseudometrics on $X$ that are uniformly continuous on $(X \times X, \mathscr{U} \times \mathscr{U})$ form a base for a uniform structure $\mathscr{D}_{\mathscr{U}}$ such that the family $\{\{(x, y): d(x, y)<\varepsilon\}: d \in \mathscr{P}, \varepsilon>0\}$ is a base for $\mathscr{U}$; in particular, $\tau_{\mathscr{U}}=\tau_{\mathscr{D}_{\mathscr{U}}}$.

Then they were proved in Section 3 of [13] the following results:

(a) If $\mathscr{D}$ is a (Hausdorff) uniform structure on a set $X$, then the family $\mathscr{B}=$ $\left\{\left(M_{d}, *\right): d \in \mathscr{D}\right\}$ is a base for a (Hausdorff) fuzzy uniform structure $\varphi_{*}(\mathscr{D})$ on $X$. Besides, $\mathscr{U}_{\mathscr{D}}=\mathscr{U}_{\varphi_{*}(\mathscr{D})}$, and thus $\tau_{\mathscr{D}}=\tau_{\varphi_{*}(\mathscr{D})}$.

(b) If $\mathscr{M}$ is a fuzzy uniform structure on $X$, choose for each $(M, *) \in(\mathscr{M}, *)$ a pseudometric $d(M)$ on $X$ whose induced uniformity coincides with $\mathscr{U}_{M}$. Then, the family $\{d(M):(M, *) \in(\mathscr{M}, *)\}$ is a subbase for a uniform structure $\psi(\mathscr{M})$ on $X$. Besides $\mathscr{U}_{\mathscr{M}}=\mathscr{U}_{\psi(\mathscr{M})}$, and thus $\tau_{\mathscr{M}}=\tau_{\psi(\mathscr{M})}$.

(c) The mappings $\Phi_{*}: \mathbf{U N I F} \rightarrow \mathbf{F U N I F}(*)$ and $\Psi: \mathbf{F U N I F}(*) \rightarrow \mathbf{U N I F}$ defined, respectively, as

$$
\begin{array}{ll}
\Phi_{*}((X, \mathscr{U}))=\left(X, \varphi_{*}\left(\mathscr{D}_{\mathscr{U}}\right), *\right) & \text { for every }(X, \mathscr{U}) \in \mathbf{U N I F}, \\
\Psi((X, \mathscr{M}, *))=\left(X, \mathscr{U}_{\psi(\mathscr{M})}\right) & \text { for every }(X, \mathscr{M}, *) \in \mathbf{F U N I F}(*)
\end{array}
$$

and both $\Phi_{*}$ and $\Psi$ leave the mappings unchanged, are (covariant) functors, and $\Phi_{*} \circ \Psi=1_{\mathbf{F U N I F}(*)}$ and $\Psi \circ \Phi_{*}=1_{\mathbf{U N I F}}$. Consequently, the categories UNIF and $\mathbf{F U N I F}(*)$ are isomorphic. In particular, $\mathbf{F U N I F}(*)$ is a topological category.

(d) Given continuous t-norms $*$ and $\star$, the categories $\mathbf{F U N I F}(*)$ and $\mathbf{F U N I F}(\star)$ are isomorphic.

Finally, we recall some notions and results on completeness and completion of fuzzy uniform structures which will needed in the rest of the paper.

Following [13, Section 4], a filter $\mathscr{F}$ on a fuzzy uniform space $(X, \mathscr{M}, *)$ is said to be a Cauchy filter if for each $M \in \mathscr{M}$, each $\varepsilon \in(0,1)$ and each $t>0$, there is $x \in X$ such that $B_{M}(x, \varepsilon, t) \in \mathscr{F}$. The fuzzy uniform space $(X, \mathscr{M}, *)$ is called complete if every Cauchy filter on $X$ converges. In this case, we say that $(\mathscr{M}, *)$ is a complete fuzzy uniform structure on $X$. A one-to-one, fuzzy uniformly continuous mapping whose inverse is also fuzzy uniformly continuous is called a fuzzy uniform isomorphism. A fuzzy completion of a fuzzy uniform space $(X, \mathscr{M}, *)$ is a pair $(f,(\widehat{X}, \widehat{\mathscr{M}}, *))$ where $(\widehat{X}, \widehat{\mathscr{M}}, *)$ is a complete fuzzy uniform space and $f$ is a fuzzy uniform isomorphism from $X$ onto a dense subspace of $(\widehat{X}, \widehat{\mathscr{M}}, *)$.

It was proved in Section 4 of [13] that the functors $\Phi_{*}$ and $\Psi$ preserve completeness, and that each Hausdorff fuzzy uniform space has a unique (up to fuzzy uniform isomorphism) Hausdorff fuzzy completion.

\section{The Fine FUZZY UnIformity AND THE STONE-ČECH FUZZY COMPACTIFICATION}

We begin by describing the fine fuzzy uniform structure. For a Tychonoff space $X$, the fine (or the universal) uniformity is generated by all the continuous pseudometrics and, consequently, it is the largest admissible uniformity on $X$. Motivated by this fact, given a continuous t-norm $*$, by the fine (or universal) fuzzy uniform 
structure on $X$ we mean the fuzzy uniform structure $(\mathscr{F} \mathscr{N}, *)$ generated by all the continuous fuzzy pseudometrics on $X$. Our purpose is to show that $(\mathscr{F} \mathscr{N}, *)$ is the largest fuzzy uniform structure on $X$.

From now on, assume that we are given a continuous t-norm, say, *. Our first result asserts that $(\mathscr{F} \mathscr{N}, *)$ is in fact a fuzzy uniform structure. We will need the following lemma. (We omit the proof which is an easy adaptation of the argument used in Proposition 1 of [23].)

LEMMA 3.1. Let $(X, \tau)$ be a topological space. If $(M, *)$ is an admissible fuzzy pseudometric on $X$, then $M$ is continuous on $X \times X \times(0, \infty)$.

THEOREM 3.2. If $(X, \tau)$ is a Tychonoff space, then the family of all fuzzy pseudometrics that are continuous on $X \times X \times(0, \infty)$ is a base for an admissible fuzzy uniform structure on $X$.

Proof. By [9, Problem 15G.4] the family, say $\mathscr{D}$, of all continuous pseudometrics on $X \times X$ is an admissible uniform structure. Thus, Proposition 3.8 in [13] shows that the family

$$
\left\{\left(M_{d}, *\right): d \in \mathscr{D}\right\}
$$

is a base for an admissible fuzzy uniform structure on $X$. Lemma 3.1 concludes the proof.

As a consequence of the previous result, $(\mathscr{F} \mathscr{N}, *)$ is an admissible fuzzy uniform structure on $X$. The following theorem states the desired property of $(\mathscr{F} \mathscr{N}, *)$.

THEOREM 3.3. If $(\mathscr{M}, *)$ is an admissible fuzzy uniform structure on $X$, then $(\mathscr{M}, *) \subseteq(\mathscr{F} \mathscr{N}, *)$.

Proof. It suffices to show that $(\mathscr{M}, *)$ has a base formed by continuous fuzzy pseudometrics on $X \times X \times(0, \infty)$. To see this, choose, for each $M \in(\mathscr{M}, *)$, a pseudometric $d(M)$ such that $\mathscr{U}_{M}=\mathscr{U}_{d(M)}$. Then $d(M)$ is continuous on $X \times X$ and the family

$$
\mathscr{B}=\left\{\left(M_{d(M)}, *\right): M \in(\mathscr{M}, *)\right\}
$$

is a base for the fuzzy uniform structure of the space $\Phi_{*}(\Psi((X, \mathscr{M}, *)))$. Since $\Phi_{*} \circ \Psi=1_{\text {FUNIF }(*)}$, we have just showed that $\mathscr{B}$ is a base for $(\mathscr{M}, *)$.

We now turn to a discussion of another kind of completion which is relevant in the framework of Tychonoff spaces: the Stone-Čech compactification. Our approach makes use of the notion of the Samuel compactification of a space $X$ with respect to a uniformity $\mathscr{U}$. Thus, we start by considering precompact fuzzy uniform structures because the idea of a Samuel compactification relies on the concept of precompactness. As we show below the relation between precompactness and compactness emerges clearly by means of the concept of completeness.

DEFINITION 3.4. A fuzzy uniform structure $(\mathscr{M}, *)$ on a set $X$ is said to be precompact if for each $\varepsilon \in(0,1)$, each $t>0$, and each fuzzy pseudometric $M \in$ $(\mathscr{M}, *)$ there is a finite subset $F$ of $X$ such that $X=\bigcup_{a \in F} B_{M}(a, \varepsilon, t)$. In this case, we say that $(X, \mathscr{M}, *)$ is a precompact fuzzy uniform space. 
It should be noted that the definition of a precompact fuzzy uniform structure, as stated in Definition 3.4, is a natural adaptation of the notion of a precompact fuzzy metric space given in [10, Definition 1].

The following simple but useful result is an easy consequence of the well-known facts that a pseudometric space $(X, d)$ is precompact if and only if every ultrafilter on $X$ is Cauchy on the uniform space $\left(X, \mathscr{U}_{d}\right)$, and that $\mathscr{U}_{d}=\mathscr{U}_{M_{d}}$.

PROPOSITION 3.5. A pseudometric space $(X, d)$ is precompact if and only if the fuzzy pseudometric space $\left(X, M_{d}, *\right)$ is precompact.

With these previous results we can obtain the following:

THEOREM 3.6. The functors $\Phi_{*}$ and $\Psi$ preserve precompactness.

Proof. Suppose that $(X, \mathscr{M}, *)$ is a precompact fuzzy uniform space. Since, by $[13$, Proposition 3.12], the uniformity of the uniform space $\Psi((X, \mathscr{M}, *))$ is $\mathscr{U}_{\mathscr{M}}$, the fact that $\Psi$ preserves precompactness is a straightforward consequence of the description of $\mathscr{U}_{\mathscr{M}}$ obtained in [13, Proposition 3.4]. Now to establish that $\Phi_{*}$ preserves precompactness, notice that if $(X, \mathscr{U})$ is a precompact uniform space, then the pseudometric spaces $(X, d)$ are precompact whenever $d$ belongs to the family $\mathscr{D}_{\mathscr{U}}$ of the all uniformly continuous pseudometrics on $(X \times X, \mathscr{U} \times \mathscr{U})$. Then by Proposition 3.5 all the fuzzy pseudometric spaces $\left(X, M_{d}, *\right)$ are precompact whenever $d \in \mathscr{D}_{\mathscr{U}}$. The results now follows from the fact that the fuzzy pseudometrics $\left(M_{d}, *\right)$ $\left(d \in \mathscr{D}_{\mathscr{U}}\right)$ generate the fuzzy uniform structure on $\Phi_{*}((X, \mathscr{U}))$.

COROLLARY 3.7. Let $\left\{\left(X_{i}, \mathscr{M}_{i}, *\right)\right\}_{i \in I}$ be a family of fuzzy uniform spaces. Then the product fuzzy uniform space $\prod_{i \in I}\left(X_{i}, \mathscr{M}_{i}, *\right)$ is precompact if and only if all $\left(X_{i}, \mathscr{M}_{i}, *\right)$ are precompact.

Proof. According to [6, Theorem 8.3.3], the uniform space $\Psi\left(\prod_{i \in I}\left(X_{i}, \mathscr{M}_{i}, *\right)\right)$ is precompact if and only if all the uniform spaces $\Psi\left(\left(X_{i}, \mathscr{M}_{i}, *\right)\right)$ are precompact. Thus, the result follows from the fact that $\Phi_{*}$ and $\Psi$ preserve precompactness, and the equality $\Phi_{*} \circ \Psi=1_{\mathbf{F U N I F}(*)}$.

COROLLARY 3.8. (a) A fuzzy uniform space $(X, \mathscr{M}, *)$ is compact if and only if it is both precompact and complete.

(b) A completion of a fuzzy uniform space $(X, \mathscr{M}, *)$ is compact if and only if $(X, \mathscr{M}, *)$ is precompact.

Proof. (a) First notice that by [13, Proposition 3.12], it follows that $(X, \mathscr{M}, *)$ is compact if and only if $\Psi((X, \mathscr{M}, *))$ is compact. Now, by [14, Theorem 32], this is equivalent to $\Psi((X, \mathscr{M}, *))$ being precompact and complete. The result is an easy consequence of the fact that both $\Phi_{*}$ and $\Psi$ preserve completeness and precompactness.

(b) Assume that $(X, \mathscr{M}, *)$ has a compact fuzzy completion $(\widehat{X}, \widehat{\mathscr{M}}, *)$. By clause (a) and the fact that $\Psi$ preserves precompactness, $\Psi((X, \mathscr{M}, *))$ is a dense subspace of the precompact uniform space $\Psi((\widehat{X}, \widehat{\mathscr{M}}, *))$. Since dense subspaces of precompact uniform spaces are precompact, $(X, \mathscr{M}, *)=\Phi_{*}(\Psi((X, \mathscr{M}, *)))$ is a precompact fuzzy uniform space. 
To see the converse, notice that a completion of the uniform space $\Psi((X, \mathscr{M}, *))$ is compact in the case that $(X, \mathscr{M}, *)$ is precompact $([14$, Theorem 32]). Then the result follows from the fact the $\Phi_{*}$ preserves completeness.

Now we will deal with compact fuzzy uniform spaces. We will see that the usual properties of compact uniform spaces also hold for compact fuzzy uniform spaces. We begin with a usual property of continuous mappings on compact fuzzy uniform spaces.

PROPOSITION 3.9. Each continuous mapping from a compact fuzzy uniform space into a fuzzy uniform space is fuzzy uniformly continuous.

Proof. Let $f:(X, \mathscr{M}, *) \rightarrow(Y, \mathscr{N}, \star)$ be a continuous mapping between two fuzzy uniform spaces with $(X, \mathscr{M}, *)$ compact. Then the uniform space $(X, \Psi((M, *)))$ is compact so that $f:(X, \Psi((M, *))) \rightarrow(X, \Psi((N, \star)))$ is uniformly continuous $([14$, Chapter 6, Proposition 31]. Now the conclusion follows from [13, Proposition 3.13].

It is well known that every Hausdorff compact space has a unique admissible uniformity. A similar result is valid for fuzzy uniform spaces. The reason is apparent: given a continuous t-norm $*$, an easy consequence of Theorem 3.6 is that there exists a one-to-one correspondence between (precompact) fuzzy uniform structures and (precompact) uniformities on a set $X$. From this fact and [9, Problem 15R], we have:

PROPOSITION 3.10. For a Hausdorff topological space $(X, \tau)$, the following are equivalent:

(i) $(X, \tau)$ has a unique admissible uniformity.

(ii) For each continuous t-norm $*,(X, \tau)$ has a unique admissible fuzzy uniform structure.

(iii) There exists a continuous t-norm * for which $(X, \tau)$ has a unique admissible fuzzy uniform structure.

(iv) For each continuous t-norm $*,(X, \tau)$ has a unique admissible precompact fuzzy uniform structure.

(v) There exists a continuous t-norm * for which $(X, \tau)$ has a unique admissible precompact fuzzy uniform structure.

COROLLARY 3.11. Let $(X, \tau)$ be a Hausdorff compact topological space. Then for each continuous t-norm $*,(X, \tau)$ has a unique admissible fuzzy uniform structure.

In the remainder of this section all spaces are assumed to be Tychonoff. We now turn our attention to coarser precompact fuzzy uniform structures and Samuel fuzzy compactifications. In his paper [27], Samuel shows that for every uniformity $\mathscr{U}$ on $X$ the uniformity $\mathscr{S}$, generated by the family of all functions from $X$ into the unit interval $\mathbb{I}$ (endowed with the usual topology) which are uniformly continuous with respect to $\mathscr{U}$ and the unique uniformity on $\mathbb{I}$, is an admissible precompact uniformity on $X$ coarser than $\mathscr{U}$. It is not difficult to prove that the uniformity $\mathscr{S}$ is the finest uniformity on $X$ which is precompact and coarser than $\mathscr{U}$. The compact space $s X$, where $(s X, \widehat{\mathscr{S}})$ is the completion of the uniform space $(X, \mathscr{S})$, is called the Samuel 
compactification of $X$ with respect to $\mathscr{U}$. Samuel's results prompt the authors to introduce the following notion of a Samuel fuzzy compactification: Let $(X, \mathscr{M}, *)$ be a fuzzy uniform space. Consider the family $\mathcal{J}$ of all fuzzy uniformly continuous functions from $(X, \mathscr{M}, *)$ into $\mathbb{I}$ equipped with its unique fuzzy uniform structure. The completion of the fuzzy uniform space $\left(X, \mathscr{S}_{\mathcal{J}}, *\right)$, where $\left(\mathscr{S}_{\mathcal{J}}, *\right)$ is the coarser of all fuzzy uniform structures $(\mathscr{N}, *)$ on $X$ enjoying the properties

(i) $(\mathscr{N}, *) \subseteq(\mathscr{M}, *)$, and

(ii) if $f \in \mathcal{J}$, then $f:(X, \mathscr{N}, *) \rightarrow \mathbb{I}$ is fuzzy uniformly continuous, is called the Samuel fuzzy compactification of $X$ with respect to $(\mathscr{M}, *)$.

In the sequel, given a function $f: X \rightarrow \mathbb{R}$, we will denote by $\psi_{f}$ the pseudometric on $X$ defined for all $x, y \in X$ as

$$
\psi_{f}(x, y)=|f(x)-f(y)| \text {. }
$$

$C^{*}(X)$ stands for the family of all bounded continuous real-valued functions on $X$. The family of all continuous functions from $X$ into $\mathbb{I}$ is denoted by $C^{*}(X, \mathbb{I})$.

THEOREM 3.12. For a fuzzy uniform space $(X, \mathscr{M}, *)$, the following are equivalent:

(i) $(X, \mathscr{M}, *)$ is precompact.

(ii) $(\mathscr{M}, *)$ is generated by a family of fuzzy pseudometrics $M_{\psi_{f}}$ with $f$ a fuzzy uniformly continuous function from $(X, \mathscr{M}, *)$ into $\mathbb{I}$.

(iii) $(\mathscr{M}, *)$ is generated by a family of fuzzy pseudometrics $M_{\psi_{f}}$ with $f \in C^{*}(X)$.

Proof. (ii) $\Rightarrow$ (iii) is obvious. So we only need to show (i) $\Rightarrow$ (ii) and (iii) $\Rightarrow(\mathrm{i})$.

(i) $\Rightarrow\left(\right.$ ii) Consider the uniform space $\left(X, \mathscr{U}_{\psi(\mathscr{M})}\right)=\Psi((X, \mathscr{M}, *))$. Since by Theorem 3.6 the functor $\Psi$ preserves precompactness, its completion $\left(\widehat{X}, \widehat{\mathscr{U}_{\psi(\mathscr{M})}}\right)$ is a compact space whose unique admissible uniformity is generated by the family $\left\{\psi_{f}: f \in C^{*}(\widehat{X}, \mathbb{I})\right\}$. Thus, the fuzzy uniformity of the space $\Phi_{*}\left(\left(\widehat{X}, \widehat{\left.\mathscr{U}_{\psi(\mathscr{M}}\right)}\right)\right)$ is generated by $\left\{M_{\psi_{f}}: f \in C^{*}(\widehat{X}, \mathbb{I})\right\}$. By [13, Theorem 4.1], $\Phi_{*}\left(\left(\widehat{X}, \widehat{\mathscr{U}_{\psi}(\mathscr{M})}\right)\right)$ is the completion of the fuzzy uniform space $(X, \mathscr{M}, *)$ which implies that $(\mathscr{M}, *)$ is generated by the fuzzy pseudometrics $M_{\psi_{f \mid}}$ with $f \in C^{*}(\widehat{X}, \mathbb{I})$. Since every continuous function on a compact space is fuzzy uniformly continuous, every function $\left.f\right|_{X}$ with $f \in C(\widehat{X}, \mathbb{I})$ is fuzzy uniformly continuous from $(X, \mathscr{M}, *)$ into $\mathbb{I}$.

(iii) $\Rightarrow$ (i) Suppose that $(\mathscr{M}, *)$ is generated by a family $\mathscr{F}$ of fuzzy pseudometrics $M_{f}$ with $f \in C^{*}(X)$. Then the family $\left\{\psi_{f}: M_{\psi_{f}} \in \mathscr{F}\right\}$ is a subbase for the uniform space $\Psi((X, \mathscr{M}, *))$ so that $\Psi((X, \mathscr{M}, *))$ is precompact $([9,15 \mathrm{I}(1)])$. The result now follows from the fact that $\Phi_{*}$ preserves precompactness (Theorem 3.6).

As straightforward consequences of the previous theorem we have:

COROLLARY 3.13. Let $(X, \mathscr{M}, *)$ be a fuzzy uniform space. Then the fuzzy uniform structure $\left(\mathscr{S}_{\mathcal{J}}, *\right)$ on $X$ is generated by the family of all fuzzy pseudometrics $M_{\psi_{f}}$ with $f \in \mathcal{J}$.

COROLLARY 3.14. The fuzzy uniform structure $\left(\mathscr{S}_{\mathfrak{J}}, *\right)$ of a space $X$ with respect to a fuzzy uniform structure $(\mathscr{M}, *)$ is the finest fuzzy uniform structure on $X$ which is precompact and coarser than $(\mathscr{M}, *)$. 
COROLLARY 3.15. The image under $\Psi$ of the Samuel fuzzy compactification of $X$ with respect to a fuzzy uniform structure $(\mathscr{M}, *)$ is the Samuel compactification of $X$ with respect to the uniformity $\mathscr{U}_{\psi(\mathscr{M})}$. Conversely, the image under $\Phi_{*}$ of the Samuel compactification of $X$ with respect to a uniformity $\mathscr{U}$ is the Samuel fuzzy compactification of $X$ with respect to the fuzzy uniform structure $\left(\varphi_{*}\left(\mathscr{D}_{\mathscr{U}}\right), *\right)$.

Next we shall introduce the Stone-Čech fuzzy compactification of a space $X$. Several constructions are known of the usual Stone-Čech compactification $\beta X$ of $X$. Probably, the simplest is the one presented in [9, Chapter 11] which makes use of an embedding in the product of real lines. The procedure adopted in this paper fits into the concept of a Samuel fuzzy compactification.

DEFINITION 3.16. The Samuel fuzzy compactification $\beta_{F} X$ of $X$ with respect to the finest fuzzy uniform structure on $X$ is called the Stone-Čech fuzzy compactification of $X$.

The following theorem provides a useful characterization of $\beta_{F} X$.

THEOREM 3.17. The Stone-Čech fuzzy compactification $\beta_{F} X$ of $X$ is the completion of the fuzzy uniform space $\left(X, C_{F}^{*}, *\right)$ where the fuzzy uniform structure $\left(C_{F}^{*}, *\right)$ is generated by the family $\left\{M_{\psi_{f}}: f \in C^{*}(X)\right\}$.

Proof. As a matter of definition, $\beta_{F} X$ is the completion of $\left(X, \mathscr{S}_{\mathcal{F}}, *\right)$ where $\left(\mathscr{S}_{\mathcal{F}}, *\right)$ is the finest precompact fuzzy uniform structure on $X$. The result now follows from Theorem 3.12 and Corollary 3.13.

COROLlARY 3.18. Let $X$ be a space. Then $\Psi\left(\beta_{F} X\right)=\beta X$ and $\Phi_{*}(\beta X)=\beta_{F} X$.

As an application of the previous results, fixed a continuous t-norm $*$, we will describe the unique fuzzy uniform structure on the Tychonoff plank, the name commonly given to Tychonoff's classic example of a nonnormal space. As is customary, the constant function, on any set, whose constant value is the real number 1 , is denoted by 1 .

EXAMPLE 3.19. Let $\mathbf{W}^{\star}$ denote the compact space of all ordinals less or equal to the first uncountable ordinal $\omega_{1}$. Consider the compact space $\mathbf{W}^{\star} \times \mathbf{N}^{\star}$ where $\mathbf{N}^{\star}$ stands for the one-point compactification $\mathbf{N} \cup\{\omega\}$ of the natural numbers $\mathbf{N}$ endowed with the discrete topology. The Tychonoff's plank $\mathbf{T}$ is the space

$$
\mathbf{T}=\left(\mathbf{W}^{\star} \times \mathbf{N}^{\star}\right) \backslash\left\{\left(\omega_{1}, \omega\right)\right\} .
$$

It is a well-known fact that $\mathbf{T}$ has a unique admissible (Hausdorff) uniformity generated by the family $\mathscr{D}$ of all the pseudometrics $\psi_{f}(f \in C(\mathbf{T}))$ where $f$ vanishes at $\left(\omega_{1}, \omega\right)$ or $f=\mathbf{1}$ (see $[9,8.20$ and Problem 15R(7)]). Thus, given a continuous t-norm $*, \mathbf{T}$ has a unique admissible fuzzy uniform structure $(\mathscr{M}, *)$. Of course, the construction of $(\mathscr{M}, *)$ may be accomplished in a variety of ways. We will explain two possible approaches to illustrating such procedures.

The first one is a fairly direct consequence of Proposition 3.8 in [13]: indeed, the fuzzy standard pseudometrics $M_{\psi_{f}}$ (with $f \in C(\mathbf{T})$ ) generate $(\mathscr{M}, *)$.

To see a second method of identifying $(\mathscr{M}, *)$, for each $\psi_{f} \in \mathscr{D}$ define a fuzzy pseudometric $M_{\psi_{f}, 01}$ as follows: 


$$
M_{\psi_{f}, 01}(x, y, 0)=0 \text { for all } x, y \in \mathbf{T},
$$

and, for all $x, y \in \mathbf{T}$ and $t>0$,

$$
M_{\psi_{f}, 01}(x, y, t)= \begin{cases}1 & \text { if } \psi_{f}(x, y) \leq t \\ 0 & \text { if } \psi_{f}(x, y)>t\end{cases}
$$

It is an easy matter to show that $\left(M_{\psi_{f}, 01}, *\right)$ is a fuzzy pseudometric on $\mathbf{T}$ whose induced topology coincides with the topology induced by the pseudometric $\psi_{f}(f \in$ $C(\mathbf{T}))$. Thus, the fuzzy uniform structure $(\mathscr{H}, *)$ generated by $\left\{\left(M_{\psi_{f}, 01}, *\right): f \in C(\mathbf{T})\right\}$ is admissible on $\mathbf{T}$. Since $\mathbf{T}$ has a unique admissible fuzzy uniform structure, $(\mathscr{H}, *)=(\mathscr{M}, *)$.

We close the section with another example of application of our techniques.

EXAMPLE 3.20. Let $*$ be a continuous t-norm. If $X$ is a discrete space, the fuzzy uniform structure $(\mathscr{M}, *)$ on $X$ generated by all $\left(M_{\psi_{f_{x}}}, *\right)$, where $f_{x}$ is the characteristic function of $\{x\}(x \in X)$, is precompact (Theorem 3.12) so that, by Corollary 3.8 , the completion $(\widehat{X}, \widehat{\mathscr{M}}, *)$ of $(X, \mathscr{M}, *)$ is compact. Since the completion of $X$ for the uniformity generated by all $\psi_{f_{x}}$ is the one-point compactification $X^{\star}$ of $X$, (see, for instance, [9, Problem 15K]), the topology of $X^{\star}$ coincides with the topology of $\widehat{X}$. Thus, the one-point compactification $X^{\star}$ of a discrete space $X$ can be described by the fuzzy completion of $(X, \mathscr{M}, *)$, that is, the completion of the fuzzy uniform structure on $X$ generated by the family of all the fuzzy pseudometric $\left(M_{\psi_{x}}, *\right)(x \in X)$ where

$$
M_{\psi_{f_{x}}}(z, y, 0)=0 \text { for all } z, y \in X
$$

and, for all $z, y \in X$ and $t>0$,

$$
M_{\psi_{f_{x}}}(z, y, t)=\left\{\begin{array}{cl}
1 & \text { if } z \neq x \text { and } y \neq x \\
1 & \text { if } z=y=x \\
\frac{t}{t+1} & \text { otherwise }
\end{array}\right.
$$

\section{FuzZy uniform structures on topological groups}

By a fuzzy uniformizable group $G$ it is meant a 4 -tuple $(G, \cdot, \mathscr{M}, *)$ where $(G, \mathscr{M}, *)$ is a fuzzy uniform space and $\left(G, \cdot \tau_{\mathscr{M}}\right)$ is a topological group. It is a well-known fact that every $T_{0}$ topological group is a Tychonoff space. Therefore a topological group $G$ is a Tychonoff space if and only it has an admissible Hausdorff fuzzy uniform structure ([13, Corollary 3.10]).

The following definition is borrowed from [25, Definition 1].

DEFINITION 4.1. A fuzzy pseudometric $(M, *)$ on an abstract (i.e. not necessarily topological) group $G$ is said to be left-invariant (resp. right-invariant) if $M(x, y, t)=$ $M(a x, a y, t)(\operatorname{resp} . M(x, y, t)=M(x a, y a, t))$ for all $a, x, y \in G$ and all $t>0$. 
Given a topological group $(G, \tau)$, an admissible fuzzy uniform structure $(\mathscr{M}, *)$ is called left-invariant if it has a base of left-invariant fuzzy pseudometrics. The definition of right-invariant fuzzy uniformity is self-explanatory. The following result can be proved in a similar way to the one in [25, Proposition 1].

THEOREM 4.2. If $(G, \tau)$ is a topological group, then there exists an one-to-one correspondence between admissible left-invariant fuzzy uniform structures on $(G, \tau)$ and right-invariant fuzzy uniform structures on $(G, \tau)$.

Now, let $(G, \tau)$ be a topological group and let $\xi(e)$ be a base for $G$ at the neutral element $e$. Every member $U$ of $\xi(e)$ determines three entourages of the diagonal:

$$
U_{L}=\left\{(x, y): x^{-1} y \in U\right\}, U_{R}=\left\{(x, y): y x^{-1} \in U\right\} \text { and } U_{B}=U_{L} \cap U_{R} .
$$

Denote by $\mathscr{U}_{L}, \mathscr{U}_{R}$ and $\mathscr{U}_{B}$, respectively, the collection of all $U_{L}, U_{R}$ and $U_{B}$, where $U \in \xi(e)$. Each of the collections $\mathscr{U}_{L}, \mathscr{U}_{R}$ and $\mathscr{U}_{B}$ defines an admissible uniformity on the topological group $(G, \tau)$, the so-called, respectively, left uniformity, right uniformity and bilateral uniformity. From now on, unless the contrary is explicitly stated, we shall work with the left uniformity $\mathscr{U}_{L}$. Note that every statement on $\mathscr{U}_{L}$ has a dual result for both $\mathscr{U}_{R}$ and $\mathscr{U}_{B}$.

Our next result generalizes [25, Theorem 3].

THEOREM 4.3. If $(G, \tau)$ is a topological group and $(\mathscr{M}, *)$ an admissible leftinvariant fuzzy uniform structure on $G$, then the uniformity $\mathscr{U}_{\mathscr{M}}$ coincides with the left uniformity $\mathscr{U}_{L}$.

Proof. Since the fuzzy uniform structure $(\mathscr{M}, *)$ is admissible, a basic neighborhood of the neutral element $e$ is $M_{t, \varepsilon}=\{y: M(e, y, t)>1-\varepsilon\}$ with $\varepsilon \in(0,1), t>0$ and $M \in(\mathscr{M}, *)$. Therefore a base for $\mathscr{U}_{L}$ is the family $\mathscr{V}$ of all sets

$$
\left\{(x, y): x^{-1} y \in M_{t, \varepsilon}\right\}, \quad(\varepsilon \in(0,1), t>0, M \in(\mathscr{M}, *)) .
$$

We conclude the proof by showing that $\mathscr{V}$ is base for $\mathscr{U}_{\mathscr{M}}$. In fact, given $\varepsilon \in(0,1)$, $t>0$ and $M \in(\mathscr{M}, *)$, we have

$$
\begin{aligned}
\left\{(x, y): x^{-1} y \in M_{t, \varepsilon}\right\} & =\left\{(x, y): M\left(e, x^{-1} y, t\right)>1-\varepsilon\right\} \\
& =\{(x, y): M(x, y, t)>1-\varepsilon\} \\
& =U_{M, t, \varepsilon},
\end{aligned}
$$

because $(\mathscr{M}, *)$ is left-invariant. Thus, the family $\mathscr{V}$ coincides with the base

$$
\left\{U_{M, t, \varepsilon}: M \in(\mathscr{M}, *), \varepsilon \in(0,1), t>0\right\}
$$

of $\mathscr{U}_{\mathscr{M}}$ and the proof is complete.

As a matter of fact, there exists only one admissible left-invariant fuzzy uniform structure on a topological group, say, $\mathscr{U}_{L}$. Given a topological group $(G, \tau)$, we will denote by $\mathscr{D}_{L}$ the family of all left-invariant pseudometrics which are uniformly continuous on $\left(G, \mathscr{U}_{L}\right)$.

THEOREM 4.4. Let $(G, \tau)$ be a topological group. If $(\mathscr{M}, *)$ is an admissible leftinvariant fuzzy uniform structure on $G$, then a base for $(\mathscr{M}, *)$ is the family of all standard fuzzy pseudometrics $\left(M_{d}, *\right)$ where $d$ runs over the family $\mathscr{D}_{L}$. 
Proof. Since Theorem 4.3 tells us that $\Psi((G, \mathscr{M}, *))=\left(G, \mathscr{U}_{L}\right)$, the result is a consequence of the fact that the family $\mathscr{D}_{L}$ is a base for the uniform structure associated to $\mathscr{U}_{L}$.

Since the functor $\Psi$ is one-to-one on objects, we have

COROLLARY 4.5. Let $(G, \tau)$ be a topological group. The left-invariant fuzzy uniform structure on $G$ generated by the family $\left\{\left(M_{d}, *\right): d \in \mathscr{D}_{L}\right\}$ is the only admissible left-invariant fuzzy uniform structure on $G$.

Let $\left(\mathscr{F}_{\mathscr{U}_{L}}, *\right)$ denote the uniform structure described in Corollary 4.5. Taking into account the previous results, $\left(\mathscr{F}_{\mathscr{U}_{L}}, *\right)$ is called the left fuzzy uniform structure on $(G, \tau)$. It is plain that $\Psi\left(G, \mathscr{F}_{\mathscr{U}_{L}}, *\right)=\left(G, \mathscr{U}_{L}\right)$.

Let $*_{L}$ denote the Lukasiewiez t-norm, i.e., $a *_{L} b=\max \{a+b-1,0\}$ for all $a, b \in[0,1]$.

EXAMPLE 4.6. Let $(G, \tau)$ be a topological group, and consider an admissible leftinvariant fuzzy uniform structure $(\mathscr{M}, *)$ on $G$, with $*_{L} \leq *$. Then, a line of reasoning similar to the one in the metric case (see [2, Remark 7.6.1]), with the trivial adjustments necessary to fit the argument to this new situation, proves that, for each $(M, *) \in(\mathscr{M}, *)$, the function $d_{M}$ defined by

$$
d_{M}(x, y)=\sup \{t \geq 0: M(x, y, t) \leq 1-t\}
$$

for all $x, y \in G$, is a (left-invariant) pseudometric on $G$. Observe that the uniformity $\mathscr{V}_{\mathscr{M}}$ generated by the family $\left\{d_{M}:(M, *) \in(\mathscr{M}, *)\right\}$ coincides with the uniformity $\mathscr{U}_{\mathscr{M}}$; in fact, for each $\varepsilon \in(0,1)$, we have

$$
\{(x, y): M(x, y, \varepsilon)>1-\varepsilon\}=\left\{(x, y): d_{M}(x, y)<\varepsilon\right\} .
$$

Since Theorem 4.3 tells us that $\mathscr{U}_{\mathscr{M}}=\mathscr{U}_{L}$, we have just proved that the left uniformity on $G$ can be generated by a family of left-invariant pseudometrics defined by means of left-invariant fuzzy pseudometrics.

A fuzzy uniform space $(X, \mathscr{M}, *)$ is said to be locally precompact if every $x \in X$ has a neighborhood $V$ such that the fuzzy uniform space $\left(V,\left.\mathscr{M}\right|_{V}, *\right)$ is precompact.

An argument similar to the one used in Theorem 3.6 permits us to obtain:

THEOREM 4.7. The functors $\Psi$ and $\Phi_{*}$ preserve local precompactness.

Recall that a topological group $(G, \tau)$ is said to be locally precompact if the uniform space $\left(G, \mathscr{U}_{L}\right)$ is locally precompact. If $\left(G, \mathscr{U}_{L}\right)$ is a locally precompact group, then its completion $\left(\widehat{G}, \widehat{\mathscr{U}}_{L}\right)$ is called the Weil completion of $(G, \tau)$ and it plays an important role in the theory of topological groups. The properties of the functors $\Psi$ and $\Phi_{*}$ allow us to introduce these concepts in the framework of fuzzy uniform spaces and to make use of fuzzy uniform structures to characterize several classes of topological groups.

We need to introduce some concepts. A topological group $(G, \tau)$ is fuzzy locally precompact if the fuzzy uniform space $\left(G, \mathscr{F}_{\mathscr{U}_{L}}, *\right)$ is locally precompact. In this case, its fuzzy completion $\left(\widehat{G},{\widehat{\mathscr{F}} \mathscr{U}_{L}}, *\right)$ is called the Weil fuzzy completion of $(G, \tau)$.

Since the functors $\Psi$ and $\Phi_{*}$ preserves completeness, the following result arises: 
THEOREM 4.8. The functors $\Psi$ and $\Phi_{*}$ preserve Weil completeness.

THEOREM 4.9. For a topological group $(G, \tau)$, the following are equivalent:

(i) $(G, \tau)$ is a (locally) precompact group.

(ii) The left-invariant fuzzy uniform structure $\left(\mathscr{F}_{\mathscr{U}_{L}}, *\right)$ is (locally) precompact.

(iii) The Weil fuzzy completion of $(G, \tau)$ is a (locally) compact space.

Proof. (i) $\Rightarrow$ (ii) Since $\Phi_{*}\left(\left(G, \mathscr{U}_{L}\right)\right)=\left(G, \mathscr{F}_{\mathscr{U}_{L}}, *\right)$, the implication follows from the fact that $\Phi_{*}$ preserves local precompactness.

(ii) $\Rightarrow$ (iii) It is a consequence of the well-known fact that the Weil completion of a (locally) precompact group $(G, \tau)$ is a (locally) compact space and Theorem 4.8.

(iii) $\Rightarrow$ (i) Since $\left(\widehat{G}, \widehat{\mathscr{F}}_{\mathscr{U}_{L}}, *\right)$ is (locally) compact, $\left(G, \mathscr{F}_{\mathscr{U}_{L}}, *\right)$ is (locally) precompact and then so is $\Psi\left(\left(G, \mathscr{F}_{\mathscr{U}_{L}}, *\right)\right)=\left(G, \mathscr{U}_{L}\right)$.

One of the most celebrated results on pseudocompact topological groups is the Comfort-Ross-van Douwen theorem [3, 5] which states that the Weil completion of a pseudocompact group is, actually, a compact topological group which coincides with its Stone-Čech compactification. This theorem was generalized for locally pseudocompact groups in [4] and for an arbitrary product of locally pseudocompact groups in [28]. These results are applied in order to obtain:

THEOREM 4.10. For a (locally) precompact group $(G, \tau)$, the following are equivalent:

(i) $(G, \tau)$ is a (locally) pseudocompact group.

(ii) The Weil fuzzy completion of $(G, \tau)$ is a (locally) compact group.

(iii) The Weil fuzzy completion of $(G, \tau)$ coincides with (the fine completion of $(G, \tau)) \beta_{F} X$.

We now shift to quotients topological groups. A celebrated result in the theory of metric topological groups states that given a (complete) metric group $G$ and a closed normal subgroup $N$, the quotient groups $G / N$ is a (complete) metrizable group. Moreover, if $N$ and $G / N$ are complete, then $G$ is complete.

One might tempted to try to extend this result to fuzzy metric spaces (in the sense of George and Veeramani). Nevertheless, as it was sshowed in [25], the natural way to define a fuzzy metric on $G / N$ does not satisfy the conditions of a fuzzy metric in George and Veeramani's sense. In the following, given a continuous t-norm *, we shall see as the functors $\Psi$ and $\Phi_{*}$ allow us to guess a suitable definition of a quotient fuzzy uniform structure on $G / N$.

DEFINITION 4.11. Let $\left(G, \cdot, \mathscr{F}_{\mathscr{U}_{L}}, *\right)$ be a fuzzy uniformizable group and let $N$ be a closed normal subgroup of $G$. The uniform structure of the fuzzy uniform space $\Phi_{*}\left(\left(G / N, \mathscr{U}_{L} / N\right)\right)$ is called the quotient fuzzy uniform structure on $G / N$.

Our next step is to obtain a reasonable description of the quotient fuzzy uniform structure which will be denoted by $\mathscr{F}_{\mathscr{U}_{L}} / N$. It is well known that if $d$ is a pseudometric on $\mathscr{D}_{L}$, then the pseudometrics $\widehat{d}$ defined as

$$
\widehat{d}(\widehat{x}, \widehat{y})=\inf \{d(x, y): x \in \widehat{x}, y \in \widehat{y}\}
$$


form a base for the uniform structure $\mathscr{U}_{L} / N$. It is an easy matter to see that the fuzzy pseudometric $M_{\widehat{d}}$ induced by $\widehat{d}$ on $G / N$ takes the values

$$
M_{\widehat{d}}(\widehat{x}, \widehat{y}, t)=\sup \left\{M_{d}(x, y, t): x \in \widehat{x}, y \in \widehat{y}\right\}
$$

for all $t>0$. Notice that $M_{\widehat{d}}$ is left-invariant whenever $\widehat{d}$ so is.

THEOREM 4.12. The family

$$
\mathscr{M}_{L}=\left\{\left(M_{\widehat{d}}, *\right): d \in \mathscr{D}_{L}\right\}
$$

is a base for the fuzzy uniform structure $\mathscr{F}_{\mathscr{U}_{L}} / N$.

Proof. It suffices to apply [13, Proposition 3.8].

As a consequence of the previous results, we have that the fuzzy uniformizable group $\left(G / N, \cdot, \mathscr{F}_{\mathscr{U}_{L}} / N, *\right)$ is metrizable if and only if $\left(G / N, \cdot, \mathscr{U}_{L} / N\right)$ so is.

COROLLARY 4.13. Let $(G, \tau)$ be a (complete) metrizable group and let $N$ be a closed normal subgroup. Then the fuzzy uniformizable group $\left(G / N, \cdot, \mathscr{F}_{\mathscr{U}_{L}} / N, *\right)$ is complete and metrizable. In addition, if $\left(N, \cdot,\left.\mathscr{F}_{\mathscr{U}_{L}}\right|_{N}, *\right)$ and $\left(G / N, \cdot, \mathscr{F}_{\mathscr{U}_{L}} / N, *\right)$ are complete, then $\left(G, \cdot, \mathscr{F}_{\mathscr{U}_{L}}, *\right)$ is complete.

\section{The Hausdorff-Bourbaki Fuzzy uniformity}

Given a uniform space $(X, \mathscr{U})$ we denote by $\mathscr{P}_{0}(X)$ the collection of all nonempty subsets of $X$ and by $\mathscr{K}_{0}(X)$ the collection of all nonempty compact subsets of $X$.

Let us recall that if $(X, \mathscr{U})$ is a uniform space and for each $U \in \mathscr{U}$ we put

$$
U_{H}=\left\{(A, B) \in \mathscr{P}_{0}(X) \times \mathscr{P}_{0}(X): B \subseteq U(A) \text { and } A \subseteq U(B)\right\}
$$

then $\left\{U_{H}: U \in \mathscr{U}\right\}$ is a base for a uniformity $\mathscr{U}_{H}$ on $\mathscr{P}_{0}(X)$ called the HausdorffBourbaki uniformity of $(X, \mathscr{U})([19])$.

Next we recall the construction of the Hausdorff fuzzy pseudometric of a fuzzy pseudometric space $(X, M, *)$.

Given $x \in X, A \in \mathscr{P}_{0}(X)$ and $t>0$, set $M(x, A, t)=\sup _{a \in A} M(x, a, t)$.

For each $A, B \in \mathscr{P}_{0}(X)$ let

$$
\begin{gathered}
M_{H}^{-}(A, B, 0)=M_{H}^{+}(A, B, 0)=0, \\
M_{H}^{-}(A, B, t)=\sup _{0<s<t} \inf _{a \in A} M(a, B, s), \quad M_{H}^{+}(A, B, t)=\sup _{0<s<t} \inf _{b \in B} M(A, b, s),
\end{gathered}
$$

for all $t>0$, and

$$
M_{H}(A, B, t)=\min \left\{M_{H}^{-}(A, B, t), M_{H}^{+}(A, B, t)\right\},
$$

for all $t \geq 0$.

Then $\left(M_{H}, *\right)$ is a fuzzy pseudometric on $\mathscr{P}_{0}(X)$, called the Hausdorff fuzzy pseudometric of $(X, M, *)$.

Now, we define the Hausdorff-Bourbaki fuzzy uniformity of a fuzzy uniform space as follows.

Let $(X, \mathscr{M}, *)$ be a fuzzy uniform space. For each $(M, *) \in(\mathscr{M}, *)$, let $\left(M_{H}, *\right)$ be the Hausdorff fuzzy pseudometric on $\mathscr{P}_{0}(X)$ of $(X, M, *)$. Then the collection

$$
\left\{\left(M_{H}, *\right):(M, *) \in(\mathscr{M}, *)\right\}
$$


is a subbase for a fuzzy uniformity $\left(\mathscr{M}_{H}, *\right)$ on $\mathscr{P}_{0}(X)$, which will be called the Hausdorff-Bourbaki fuzzy uniformity of $(X, \mathscr{M}, *)$.

We wish to show that actually $\mathscr{U}_{\mathscr{M}_{H}}=\left(\mathscr{U}_{\mathscr{M}}\right)_{H}$ on $\mathscr{P}_{0}(X)$ (recall that $\left(\mathscr{U}_{\mathscr{M}}\right)_{H}$ is the Hausdorff-Bourbaki uniformity on $\mathscr{P}_{0}(X)$ of the uniform space $\left(X, \mathscr{U}_{\mathscr{M}}\right)$.

To this end, we first note that if $\left\{\mathscr{U}_{i}: i \in I\right\}$ is a family of uniformities on a set $X$, then we easily deduce the following:

Fact 1. $\left(\bigvee_{i \in I} \mathscr{U}_{i}\right)_{H}=\bigvee_{i \in I}\left(\mathscr{U}_{i}\right)_{H}$

Next we generalize [23, Theorem 2] to our context as follows:

LEMMA 5.1. Let $(X, M, *)$ be a fuzzy pseudometric space. Then $\left(\mathscr{U}_{M}\right)_{H}=\mathscr{U}_{M_{H}}$ on $\mathscr{P}_{0}(X)$.

Proof. For each $n \in \mathbb{N}$ set $U_{n}=\left\{(x, y): d(x, y)<2^{-n}\right\}$. Then, for each $n \in \mathbb{N}$, we easily obtain the following relations

$$
\begin{aligned}
& \left\{(A, B) \in \mathscr{P}_{0}(X) \times \mathscr{P}_{0}(X): B \subseteq U_{n+1}(A) \text { and } A \subseteq U_{n+1}(B)\right\} \\
& \quad \subseteq\left\{(A, B) \in \mathscr{P}_{0}(X) \times \mathscr{P}_{0}(X): M_{H}(A, B, 1 / n)>1-1 / n\right\} \\
& \quad \subseteq\left\{(A, B) \in \mathscr{P}_{0}(X) \times \mathscr{P}_{0}(X): B \subseteq U_{n}(A) \text { and } A \subseteq U_{n}(B\} .\right.
\end{aligned}
$$

We conclude that $\left(\mathscr{U}_{M}\right)_{H}=\mathscr{U}_{H_{M}}$ on $\mathscr{P}_{0}(X)$.

Observe that from [13, Remark 3.6] we deduce the following:

Fact 2. $\mathscr{U}_{\mathscr{M}_{H}}=\bigvee_{M \in \mathscr{M}} \mathscr{U}_{M_{H}}$.

Fact 3. $\left(\mathscr{U}_{\mathscr{M}}\right)_{H}=\left(\bigvee_{M \in \mathscr{M}} \mathscr{U}_{M}\right)_{H}$.

Now we prove the promised result.

THEOREM 5.2. Let $(X, \mathscr{M}, *)$ be a fuzzy uniform space. Then $\mathscr{U}_{\mathscr{M}_{H}}=\left(\mathscr{U}_{\mathscr{M}}\right)_{H}$ on $\mathscr{P}_{0}(X)$.

Proof. By applying successively Fact 2, Lemma 5.1, Fact 1 and Fact 3, we obtain

$$
\mathscr{U}_{\mathscr{M}_{H}}=\bigvee_{M \in \mathscr{M}} \mathscr{U}_{M_{H}}=\bigvee_{M \in \mathscr{M}}\left(\mathscr{U}_{M}\right)_{H}=\left(\bigvee_{M \in \mathscr{M}} \mathscr{U}_{M}\right)_{H}=\left(\mathscr{U}_{\mathscr{M}}\right)_{H},
$$

on $\mathscr{P}_{0}(X)$.

Michael proved in [19] that a uniform space $(X, \mathscr{U})$ is precompact if and only if $\left(\mathscr{P}_{0}(X), \mathscr{U}_{H}\right)$ is precompact, and Morita proved in [20] (see also [1]) that a Hausdorff uniform space $(X, \mathscr{U})$ is complete if and only if $\left(\mathscr{K}_{0}(X), \mathscr{U}_{H}\right)$ is complete.

With the help of Theorem 5.2 and the following lemma, Michael's theorem and Morita's theorem can be easily extended to the fuzzy uniform framework as we shall show in the sequel.

LEMMA 5.3. (Cf. [13, Theorem 4.14 and Corollary 4.4]) Let $(X, \mathscr{M}, *)$ be a fuzzy uniform space. Then

(a) $(X, \mathscr{M}, *)$ is precompact if and only if the uniform space $\left(X, \mathscr{U}_{\mathscr{M}}\right)$ is precompact.

(b) $(X, \mathscr{M}, *)$ is complete if and only if the uniform space $\left(X, \mathscr{U}_{\mathscr{M}}\right)$ is complete.

THEOREM 5.4. Let $(X, \mathscr{M}, *)$ be a fuzzy uniform space. Then $\left(\mathscr{P}_{0}(X), \mathscr{M}_{H}, *\right)$ is precompact if and only if $(X, \mathscr{M}, *)$ is precompact. 
Proof. By Lemma 5.3 (a) and Michael's theorem, we have that $(X, \mathscr{M}, *)$ is precompact if and only if $\left(\mathscr{P}_{0}(X),\left(\mathscr{U}_{\mathscr{M}}\right)_{H}\right)$ is precompact. Since, by Theorem 5.2, $\left(\mathscr{U}_{\mathscr{M}}\right)_{H}=\mathscr{U}_{\mathscr{M}_{H}}$ on $\mathscr{P}_{0}(X)$, it follows from Lemma $5.3(\mathrm{a})$, that $\left(\mathscr{P}_{0}(X), \mathscr{M}_{H}, *\right)$ is precompact if and only if $\left(\mathscr{P}_{0}(X),\left(\mathscr{U}_{\mathscr{M}}\right)_{H}\right)$ is precompact. Thus $\left(\mathscr{P}_{0}(X), \mathscr{M}_{H}, *\right)$ is precompact if and only if $(X, \mathscr{M}, *)$ is precompact.

THEOREM 5.5. Let $(X, \mathscr{M}, *)$ be a Hausdorff fuzzy uniform space. Then $\left(\mathscr{K}_{0}(X), \mathscr{M}_{H}, *\right)$ is complete if and only if $(X, \mathscr{M}, *)$ is complete.

Proof. By Lemma 5.3 (b) and Morita's theorem, we have that $(X, \mathscr{M}, *)$ is complete if and only if $\left(\mathscr{K}_{0}(X),\left(\mathscr{U}_{\mathscr{M}}\right)_{H}\right)$ is complete. Since, by Theorem 5.1, $\left(\mathscr{U}_{\mathscr{M}}\right)_{H}=\mathscr{U}_{\mathscr{M}_{H}}$ on $\mathscr{K}_{0}(X)$, it follows from Lemma $5.3(\mathrm{~b})$, that $\left(\mathscr{K}_{0}(X), \mathscr{M}_{H}, *\right)$ is complete if and only if $\left(\mathscr{K}_{0}(X),\left(\mathscr{U}_{\mathscr{M}}\right)_{H}\right)$ is complete. Thus $\left(\mathscr{K}_{0}(X), \mathscr{M}_{H}, *\right)$ is complete if and only if $(X, \mathscr{M}, *)$ is complete.

\section{FUZZY QUASI-UNIFORM STRUCTURES}

It is well known that quasi-uniform spaces provide a natural approach to the study of point-set topology, as well as to discuss and to solve problems in theory of hyperspaces and in asymmetric topological algebra, while quasi-metric spaces constitute a useful tool in domain theory and in theoretical computer science (see, for instance, $[16,17]$ and their bibliographies).

These facts together with the recent contributions to the development of the theory of fuzzy quasi-metric spaces and their applications ([2, 11, 24, 26, etc]) motivate the natural question of extending our results on fuzzy uniform structures to the quasi-uniform setting. In this section we briefly discuss this question. In fact, we will show that a suitable combination of our techniques with well-known results on quasi-uniform spaces and fuzzy quasi-pseudometric spaces, provide indeed appropriate extensions of our main constructions.

Our basic references for quasi-uniform spaces and quasi-metric spaces are [7, 16], and for fuzzy quasi-pseudometric spaces [2, 11, 24]. Terms and undefined concepts may be found in such references.

First we give the quasi-uniform counterpart of the notion of a uniform structure.

DEFINITION 6.1. A quasi-uniform structure on a set $X$ is a nonempty family $\mathscr{D}$ of quasi-pseudometrics on $X$ such that: (i) if $d, e \in \mathscr{D}$, then $d \vee e \in \mathscr{D}$; and (ii) if $e$ is a quasi-pseudometric on $X$ and if for each $\varepsilon>0$, there exist $d \in \mathscr{D}$ and $\delta>0$ such that $d(x, y) \leq \delta$ implies $e(x, y) \leq \varepsilon$, for all $x, y \in X$, then $e \in \mathscr{D}$.

A quasi-uniform structure $\mathscr{D}$ is called $T_{0}$ if for each $x, y \in X$, with $x \neq y$, there exists $d \in \mathscr{D}$ such that $d(x, y)>0$ or $d(y, x)>0$.

The notions of base and subbase for a quasi-uniform structure are defined as in the uniform case. In particular, a nonempty family $\mathscr{D}$ of quasi-pseudometrics on $X$ satisfying condition (i) above is called a quasi-gauge in [21] and it is a base for a quasi-uniform structure on $X$.

Each quasi-uniform structure $\mathscr{D}$ on $X$ induces a topology $\tau_{\mathscr{D}}$ on $X$ such that for each $x \in X$, the family $\left\{B_{d}(x, \varepsilon): d \in \mathscr{D}, \varepsilon>0\right\}$ is a neighborhood base at $x$. If $\mathscr{D}$ is a $T_{0}$ quasi-uniform structure, then $\tau_{\mathscr{D}}$ is a $T_{0}$ topology. 
Extending classical results on uniforms structures (compare Section 2) to the quasi-uniform setting, Reilly proved in [22, Proposition 2] that a pseudometric $d$ on $X$ is quasi-uniformly continuous on $\left(X \times X, \mathscr{U} \times \mathscr{U}^{-1}\right)$ if and only if the set $\{(x, y): d(x, y)<\varepsilon\}$ belongs to $\mathscr{U}$ for all $\varepsilon>0$.

By means of this result he deduced ([22, Theorem 1$])$ that if $\mathscr{U}$ is a quasi-uniformity on $X$, then the family $\mathscr{P}$ of all quasi-pseudometrics that are quasi-uniformly continuous on $\left(X \times X, \mathscr{U} \times \mathscr{U}^{-1}\right)$ form a base for a quasi-uniform structure $\mathscr{D}_{\mathscr{U}}$ such that the family $\{\{(x, y): d(x, y)<\varepsilon\}: d \in \mathscr{P}, \varepsilon>0\}$ is a base for $\mathscr{U}$ and $\tau_{\mathscr{U}}=\tau_{\mathscr{D}_{\mathscr{U}}}$. Conversely, if $\mathscr{D}$ is a quasi-uniform structure then the family $\{\{(x, y): d(x, y)<\varepsilon\}$ : $d \in \mathscr{D}, \varepsilon>0\}$ is a base for a quasi-uniformity $\mathscr{U}_{\mathscr{D}}$ such that $\tau_{\mathscr{D}}=\tau_{\mathscr{U}}$.

Concerning fuzzy quasi-metric spaces we recall that a fuzzy quasi-pseudometric on a set $X$ is a pair $(M, *)$ where $*$ is a continuous t-norm and $M$ is a fuzzy set in $X \times X \times[0, \infty)$ that satisfies conditions (i), (ii), (iv) and (v) of Definition 2.1. If, in addition, $M$ satisfies (ii') $x=y$ if and only if $M(x, y, t)=M(y, x, t)=1$ for all $t>0$, then $(M, *)$ is called a fuzzy quasi-metric.

Note that if $(M, *)$ is a fuzzy quasi-(pseudo)metric and $M^{i}(x, y, t)=M(x, y, t) \wedge$ $M(y, x, t)$ for each $x, y \in X$ and $t \geq 0$, then $\left(M^{i}, *\right)$ is a fuzzy (pseudo)metric.

By a fuzzy quasi-(pseudo)metric space we mean a triple $(X, M, *)$ such that $X$ is a (nonempty) set and $(M, *)$ is a fuzzy quasi-(pseudo)metric.

Furthermore (cf. [11]) if, in Example 2.2, $(X, d)$ is a quasi-(pseudo)metric space, then $\left(M_{d}, *\right)$ is a fuzzy quasi-(pseudo)metric for all continuous t-norm $*$, the so-called fuzzy quasi-(pseudo)metric induced by $(X, d)$, or the standard fuzzy (pseudo)metric of $(X, d)$. The quasi-uniformities $\mathscr{U}_{d}$ and $\mathscr{U}_{M_{d}}$, induced, respectively, by $d$ and $\left(M_{d}, *\right)$, coincide and thus the topologies induced by $d$ and $\left(M_{d}, *\right)$ are the same.

Next we extend the notion of a fuzzy uniform structure to the quasi-uniform setting.

DEFINITION 6.2. Let $X$ be a set and let $*$ be a continuous t-norm. A nonempty family $(\mathscr{M}, *)$ of fuzzy quasi-pseudometrics $(M, *)$ on $X$ that satisfies conditions (FU1) and (FU2) in Definition 2.3 is called a fuzzy quasi-uniform structure for $*$.

A fuzzy quasi-uniform structure $(\mathscr{M}, *)$ is said to be $T_{0}$ if

(FU3') for each $x, y \in X$ with $x \neq y$, there exist $(M, *) \in(\mathscr{M}, *)$ and $t>0$ such that $M^{i}(x, y, t)<1$.

By a $\left(T_{0}\right)$ fuzzy quasi-uniform space we mean a triple $(X, \mathscr{M}, *)$ such that $X$ is a set and $(\mathscr{M}, *)$ is a $\left(T_{0}\right)$ fuzzy uniform structure.

The notions of subbase and base are defined as in the case of fuzzy uniform structures.

Each fuzzy quasi-uniform structure $(\mathscr{M}, *)$ on a set $X$ induces a topology $\tau_{\mathscr{M}}$ on $X$ such that for each $x \in X$, the family $\left\{B_{M}(x, \varepsilon, t): M \in \mathscr{M}, \varepsilon \in(0,1), t>0\right\}$ is a neighborhood base at $x$.

The following quasi-uniform versions of Propositions 3.8 and 3.12 of [13], respectively, are straightforward.

PROPOSITION 6.3. If $\mathscr{D}$ is a $\left(T_{0}\right)$ quasi-uniform structure on $X$, then the family $\mathscr{B}=\left\{\left(M_{d}, *\right): d \in \mathscr{D}\right\}$ is a base for a $\left(T_{0}\right)$ fuzzy quasi-uniform structure $\varphi_{*}(\mathscr{D})$. Besides, $\mathscr{U}_{\mathscr{D}}=\mathscr{U}_{\varphi_{*}(\mathscr{D})}$, and thus $\tau_{\mathscr{D}}=\tau_{\varphi_{*}(\mathscr{D})}$. 
PROPOSITION 6.4. If $\mathscr{M}$ is a fuzzy quasi-uniform structure on $X$, and for each $(M, *) \in(\mathscr{M}, *), d(M)$ is a quasi-pseudometric whose induced quasi-uniformity coincides with $\mathscr{U}_{M}$, then the family $\{d(M):(M, *) \in(\mathscr{M}, *)\}$ is a subbase for a quasi-uniform structure $\psi(\mathscr{M})$. Besides $\mathscr{U}_{\mathscr{M}}=\mathscr{U}_{\psi(\mathscr{M})}$, and thus $\tau_{\mathscr{M}}=\tau_{\psi(\mathscr{M})}$.

Now generalizing in the obvious manner the notion of a fuzzy uniformly continuous mapping to the fuzzy quasi-uniform framework we can obtain the corresponding analogues to [13, Proposition 3.11 and 3.13], and we can define the category FQUNIF of fuzzy quasi-uniform spaces and fuzzy quasi-uniformly continuous mappings.

Next, given a t-norm $*$, we shall deduce that the subcategory FQUNIF $(*)$ of fuzzy quasi-uniform spaces $(X, \mathscr{M}, *)$, is isomorphic to the category QUNIF of quasi-uniform spaces (and quasi-uniformly continuous mappings) by means of a (covariant) functor which leaves mappings unchanged. To this end define the mappings $q \Phi_{*}:$ QUNIF $\rightarrow$ FQUNIF $(*)$ and $q \Psi:$ FQUNIF $(*) \rightarrow$ QUNIF, respectively, as

$$
\begin{array}{lll}
q \Phi_{*}((X, \mathscr{U}))=\left(X, \varphi_{*}\left(\mathscr{D}_{\mathscr{U}}\right), *\right) & \text { for every } & (X, \mathscr{U}) \in \mathbf{Q U N I F}, \\
q \Psi((X, \mathscr{M}, *))=\left(X, \mathscr{U}_{\psi(\mathscr{M})}\right) & \text { for every } & (X, \mathscr{M}, *) \in \mathbf{F Q U N I F}(*) .
\end{array}
$$

and both $q \Phi_{*}$ and $q \Psi$ leave the mappings unchanged.

Similarly to [13, Theorem 3.14 and Cor, 3.15] we can obtain the following:

THEOREM 6.5. $q \Phi_{*}$ and $q \Psi$ are covariant functors, $q \Phi_{*} \circ q \Psi=1_{\mathbf{F Q U N I F}(*)}$ and $q \Psi \circ$ $q \Phi_{*}=1_{\mathrm{QUNIF}}$. Hence, the categories $\mathbf{Q U N I F}$ and $\mathbf{F Q U N I F}(*)$ are isomorphic.

Finally, we discuss bicompleteness and bicompletion of fuzzy quasi-uniform structures.

First note that if $(X, \mathscr{M}, *)$ is a $\left(T_{0}\right)$ fuzzy quasi-uniform space, then $\left(X, \mathscr{M}^{i}, *\right)$ is a (Hausdorff) fuzzy uniform space, where by $\mathscr{M}^{i}$ we denote the family of fuzzy pseudometrics $\left(M^{i}, *\right)$ when $(M, *) \in(\mathscr{M}, *)$.

We say that a fuzzy quasi-uniform space $(X, \mathscr{M}, *)$ is bicomplete if the fuzzy uniform space $\left(X, \mathscr{M}^{i}, *\right)$ is complete. In this case, we say that $(\mathscr{M}, *)$ is a bicomplete fuzzy quasi-uniform structure on $X$.

As in [13, Theorem 4.1], we can show that $q \Phi_{*}$ and $q \Psi$ preserve bicompleteness.

A one-to-one fuzzy quasi-uniformly continuous mapping whose inverse is also fuzzy quasi-uniformly continuous is called a fuzzy quasi-isomorphism. A fuzzy bicompletion of a fuzzy quasi-uniform space $(X, \mathscr{M}, *)$ is a pair $(f,(\widehat{X}, \widehat{\mathscr{M}}, *))$ where $(\widehat{X}, \widehat{\mathscr{M}}, *)$ is a bicomplete fuzzy quasi-uniform space and $f$ is a fuzzy quasiisomorphism from $X$ onto a dense subspace of $\left(\widehat{X},(\widehat{\mathscr{M}})^{i}, *\right)$.

It is well known that every $T_{0}$ quasi-uniform space has a $T_{0}$ bicompletion which is unique up to quasi-isomorphism (the construction of the bicompletion may be found in [7, Chapter 3]). With the help of this result, we can extend Theorem 4.3 of [13] as follows.

THEOREM 6.6. Each $T_{0}$ fuzzy quasi-uniform space has a unique (up to quasiisomorphism) $T_{0}$ fuzzy bicompletion.

\section{REFERENCES}

[1] G. Artico and R. Moresco, Notes on the topologies and uniformities of hyperspaces, Rend. Sem. Mat. Univ. Padova 63 (1980) 51-60. MR 605782 (82h:54041) 
[2] Y. J. Cho, M. Grabiec, and V. Radu, On Nonsymmetric Topological and Probabilistic Structures, Nova Science Publishers Inc., New York, 2006. MR 2329748 (2008c:54001)

[3] W. W. Comfort and K. A. Ross, Pseudocompactness and uniform continuity in topological groups, Pacific J. Math. 16 (1966) 483-496. MR 0207886 (34 \#7699)

[4] W. W. Comfort and F. J. Trigos-Arrieta, Locally pseudocompact topological groups, Topology Appl. 62 (1995) 263-280. MR 1326826 (96c:22002)

[5] E. K. van Douwen, Homogeneity of $\beta G$ if $G$ is a topological group, Colloq. Math. 41 (1979) 193-199. MR 591923 (82a:22004)

[6] R. Engelking, General Topology, second ed., Sigma Series in Pure Mathematics, vol. 6, Heldermann Verlag, Berlin 1989. MR 1039321 (91c:54001)

[7] P. Fletcher and W.F. Lindgren, Quasi-Uniform Spaces, Lecture Notes in Pure and Applied Mathematics, vol. 77, Marcel Dekker Inc., New York, 1982. MR 660063 (84h:54026)

[8] A. George and P. Veeramani, On some results in fuzzy metric spaces, Fuzzy Sets and Systems 64 (1994) 395-399. MR 1289545 (95e:54010)

[9] L. Gillman and M. Jerison, Rings of Continuous Functions, Springer-Verlag, New York, 1976, Reprint of the 1960 edition, Graduate Texts in Mathematics, No. 43. MR 0407579 (53 \#11352)

[10] V. Gregori and S. Romaguera, Some properties of fuzzy metric spaces, Fuzzy Sets and Systems 115 (2000) 485-489. MR 1781550 (2001e:54008)

[11]_Fuzzy quasi-metric spaces, Appl. Gen. Topol. 5 (2004) 129-136. MR 2087287

[12] G. Jäger, Compactification of lattice-valued convergence spaces, Fuzzy Sets and Systems 161 (2010) 1002-1010. MR 2592438

[13] J. Gutiérrez García, S. Romaguera, and M. Sanchis, Fuzzy uniform structures and continuous t-norms, Fuzzy Sets and Systems 161 (2010) 1011-1021.

[14] J. L. Kelley, General Topology, Springer-Verlag, New York, 1975, Reprint of the 1955 edition [Van Nostrand, Toronto, Ont.], Graduate Texts in Mathematics, No. 27. MR 0370454 (51 \#6681)

[15] I. Kramosil and J. Michálek,Fuzzy metrics and statistical metric spaces, Kybernetika (Prague) 11 (1975) 336-344. MR 0410633 (53 \#14381)

[16] H.-P. A. Künzi,Nonsymmetric distances and their associated topologies: about the origins of basic ideas in the area of asymmetric topology, Handbook of the history of general topology, Vol. 3, Hist. Topol., 3, Kluwer Acad. Publ., Dordrecht, 2001, pp. 853-968. MR 1900267 (2003d:54001)

[17] H.-P. A. Künzi,An introduction to quasi-uniform spaces, Beyond topology, 239-304, Contemp. Math., 486, Amer. Math. Soc., Providence, RI, 2009. MR 2521946

[18] Y.M. Liu and M.K. Luo, Fuzzy Stone-ech-type compactifications, Fuzzy Sets and Systems 33 (1989) 355-372. MR 1033881 (91b:54010

[19] E. Michael,Topologies on spaces of subsets, Trans. Amer. Math. Soc. 71 (1951) 152-182. MR 0042109 (13,54f)

[20] K. Morita, Completion of hyperspaces of compact subsets and topological completion of openclosed maps, General Topology and Appl. 4 (1974) 217-233. MR 0350683 (50 \#3175)

[21] I. L. Reilly, Quasi-gauge spaces, J. London Math. Soc. (2) 6 (1973) 481-487. MR 0317284 (47 \#5831)

[22] I. L. Reilly, On generating quasi uniformities, Math. Ann. 189 (1970) 317-318. MR 0276923 (43 \#2663)

[23] J. Rodríguez-López and S. Romaguera, The Hausdorff fuzzy metric on compact sets, Fuzzy Sets and Systems 147 (2004) 273-283. MR 2089291

[24] J. Rodríguez-López, S. Romaguera, and J. M. Sánchez-Álvarez, The Hausdorff fuzzy quasi-metric, Fuzzy Sets and Systems 161 (2010) 1078-1096. MR 2595255 doi:10.1016/j.fss.2009.09.019.

[25] S. Romaguera and M. Sanchis, On fuzzy metric groups, Fuzzy Sets and Systems 124 (2001) 109-115. MR 1859783 
[26] S. Romaguera, A. Sapena, and P. Tirado, The Banach fixed point theorem in fuzzy quasimetric spaces with application to the domain of words, Topology Appl. 154 (2007) 2196-2203. MR 2324931 (2008b:54010)

[27] P. Samuel, Ultrafilters and compactification of uniform spaces, Trans. Amer. Math. Soc. 64 (1948) 100-132. MR 0025717 (10,54a)

[28] M. Sanchis, Continuous functions on locally pseudocompact groups, Topology Appl. 86 (1998) 5-23. MR 1619340 (99d:22006)

[29] B. Schweizer and A. Sklar, Statistical metric spaces, Pacific J. Math. 10 (1960) 313-334. MR 0115153 (22 \#5955)

Current address: Departamento de Matemáticas, Universidad del País Vasco-Euskal Herriko Unibertsitatea, Apartado 644, 48080, Bilbao, (Spain)

E-mail address: javier gutierrezgarcia@ehu.es

Current address: Instituto Universitario de Matemática Pura y Aplicada, Universidad Politécnica de Valencia, Camino de Vera s/n, 46022, Valencia (Spain)

E-mail address: sromague@mat.upv.es

Current address: Institut Universitari de Matemàtiques i Aplicacions de Castelló (IMAC), Universitat Jaume I, Campus del Riu Sec. s/n, 12071 Castelló (Spain)

E-mail address: sanchis@mat.uji.es 